\title{
Supported self-management for COPD: making progress, but there are still challenges
}

\author{
Hilary Pinnock , Liz Steed ${ }^{2}$ and Rachel Jordan ${ }^{3}$
}

Affiliations: ${ }^{1}$ Allergy and Respiratory Research Group, Usher Institute for Population Health Sciences and Informatics, The University of Edinburgh, Edinburgh, UK. ${ }^{2}$ Centre for Public Health and Primary Care, Queen Mary University of London, London, UK. ${ }^{3}$ Institute of Applied Health Research, College of Medical and Dental Sciences, University of Birmingham, Birmingham, UK.

Correspondence: Hilary Pinnock, Usher Institute for Population Health Sciences and Informatics, The University of Edinburgh, Doorway 3, Medical School, Teviot Place, Edinburgh EH8 9AG, UK. E-mail: hilary.pinnockded.ac.uk

@ERSpublications

Self-management is not a time-limited intervention but on-going support to help a person live with their condition(s) http://ow.ly/10luZq

Respiratory specialists familiar with the success of supported self-management in asthma [1] may be excused for feeling disappointed by the evolution of evidence in chronic obstructive pulmonary disease (COPD). The promise of early studies [2-4] was seemingly dashed by a series of negative trials $[5,6]$ and a trial was even terminated early for fear of a detrimental effect [7]. The most recent Cochrane review, however, concludes that interventions to support self-management reduce respiratory-related and all-cause admissions, reduce dyspnoea and improve quality of life [8]. The interventions in the included studies were heterogeneous and complex; and it was not possible to identify the individual components of supported self-management responsible for effective outcomes. A wider review of trial-level data was also unable to identify the most important components [9]. The study by JONKMAN et al. [10] in this issue of the European Respiratory Journal, which explores the characteristics of effective self-management interventions in an individual patient data meta-analysis, is therefore a useful contribution to the literature.

\section{Difficulty identifying early exacerbations}

In retrospect, the clues that self-management in COPD would be more challenging than in asthma were already available. From the health service perspective, one of the main goals of self-management is to enable patients to recognise that their condition is deteriorating and take timely, appropriate action that can prevent further development of an exacerbation and reduce the risk of an admission. However, qualitative studies in people with COPD highlight the difficulty people have in distinguishing "bad days" from the onset of an exacerbation, observing that it is "only afterwards that you know you are not well" [11]. When asked about the symptoms that indicate an exacerbation, many patients describe nebulous symptoms of "just feeling rough" [12], an observation that will resonate with any general practitioner who has been asked to visit a COPD patient in the throes of an exacerbation whose complaint is no more specific than "unwell". Analysis of telemonitoring data suggests that it is the minority of patients who can log discrete episodes of increased breathlessness, cough and purulent sputum [13]. Most experience a rolling pattern of on-going symptoms with little clarity on when treatment is appropriate. This difficulty in distinguishing the onset of an exacerbation may be one reason for the negative findings of BuCKNELL et al. [6] in their trial of self-management. They observed that only $42 \%$ of participants were "successful self-managers" and, in a post hoc analysis, showed that the intervention was effective in this sub-group. Calverley et al. [14] predicted this difficulty a decade ago from analysis of diary cards from trial participants. Despite an increase in mean symptom scores, on an individual patient basis concurrence between an exacerbation event and a change in breathless, chest tightness, cough and night wakening was evident in less than half of

Received: March 202016 | Accepted: March 212016

Conflict of interest: Disclosures can be found alongside the online version of the article at erj.ersjournals.com

Copyright CERS 2016 
the patients. Effective self-management in COPD must go beyond symptom recognition and treatment response.

\section{Living with COPD}

People with any long-term condition, even if they attend regular reviews with their healthcare professionals, are responsible for their own care for the majority of the time; they are de facto "self-managing". For the patient this is more than a narrow biomedical approach to detecting and managing exacerbations. A commonly cited definition of self-management includes having the confidence to deal not only with medical management (the focus of the cited systematic reviews [8-10]), but also the role and emotional management of their condition(s) [15]. For people living with a chronic disabling condition such as severe COPD, coping with day-to-day activities (e.g. walking, dressing and eating) may be the priority self-management skills. Support for changing health behaviours (sustained smoking cessation and increased activity) is crucial. This resonates with successful self-management interventions in other chronically disabling conditions (e.g. stroke), that focus on strategies for enabling and improving behaviours and activities of daily living rather than crisis management [16, 17]. Qualitative work has highlighted how people with severe COPD struggle with (and adapt to) the change in roles imposed by their condition [18]. Despite being aware of their difficulties, they fear being seen as "needy" and will eschew professional care in favour of (reluctantly) seeking the help of family/friends to enable them to continue living independently [19]. Self-care in this broader sense is a key motivator for many people with long-term conditions and may be harnessed to encourage engagement in the more clinical model of self-management described in systematic reviews.

Underlying these broader aspects of self-management are the individual's illness and treatment beliefs, which have consistently been shown to be important predictors of quality of life and disability [20]. Identifying and responding to beliefs (such as low sense of control, inappropriate attribution of symptoms, and high emotional perceptions) should therefore be central to self-management support.

\section{On-going support for self-management}

Taxonomies of self-management support derived from studies in a range of long-term conditions have identified components reflected in the COPD self-management literature [17]. JONKMAN et al. [10] sought to identify components of the interventions associated with a positive outcome and concluded that only the duration of the intervention showed consistent associations with all-cause admissions. The longer the intervention was supported by professional review (range 1 day to 24 months) the greater the reduction in admissions. This resonates with the findings of the meta-analysis from JORDAN et al. [9] which suggested that interventions that were more supported by healthcare professionals improved quality of life and reduced admissions. Proponents of self-management will interpret this as a dose response to the self-management intervention; cynics may speculate that the active ingredient was the professional review rather than the self-management. The truth probably lies in a combination of these explanations. Self-management is not a single event; for behaviour change to be maintained continued support is crucial. We observed the gradual evolution of self-management skills in the qualitative evaluation of "Light Touch" (a COPD self-monitoring service supported by NHS Lothian) in which the daily professional oversight of telemonitoring was replaced by access to a helpline; over 6 months patients gradually gained confidence in managing their own condition [21].

\section{The challenge of multimorbidity}

The final challenge for COPD self-management is that the majority of patients have comorbidity and half will have multiple morbidities [22], some of which may be a higher priority for them than their COPD. Common comorbidities are coronary heart disease, depression and anxiety, and pain [22]; any of which may limit day-to-day activities and mask symptoms of deterioration. There is no clear guidance on self-management advice appropriate to patients with multimorbidity. Demands from different conditions may conflict or confuse and individually tailored professional advice may be needed to help people with multimorbidity live optimally with their conditions and, more specifically, interpret and respond to deteriorating status.

\section{A whole systems approach to supporting self-management}

The conclusions of Jonkman et al. [10] remind us that supported self-management is not a time-limited intervention delivered as a once and for all package. It is not a "thing" to be given to a person with COPD (or any long-term condition) but an on-going process of joint understanding, reviewing and problem solving so that advice can be refined to help a person live better with their condition(s). This needs a whole systems approach for effective implementation $[1,23]$. Responding to patients' beliefs and priorities, provision of information and resources are essential (in our work a key request from people with COPD was for information about community resources and self-help agencies [24]) with support tailored to the 
individual's clinical, demographic and psychosocial characteristics. Practitioners need not only to be knowledgeable about COPD but also skilled in patient-centred consulting. Crucially it demands that the healthcare system is configured to: 1) support self-management; 2) address the multimorbidities with which the patient is living; 3) provide flexible access to professional advice [25]; and 4) ideally ensure continuity of care [26].

In practical terms, this will have to be achieved within the routines of existing healthcare services and the available resources. Self-management is currently supported within holistic pulmonary rehabilitation programmes (though access and uptake are challenging [27]); strong primary care systems have the potential to facilitate on-going support; patient charities could contribute to the broader aspects of living with COPD; and in the future technological approaches to care delivery may improve efficiency.

\section{Conclusion}

The individual patient data meta-analysis of Jonkman et al. [10] adds support to the increasing evidence that supported self-management can reduce admissions [8,9], and highlights that interventions involving on-going professional support were more effective [10]. There are, however, significant unresolved challenges for patients, professionals and healthcare systems. Clinical "action plans" do not reflect the difficulties patients have in identifying the onset of an exacerbation and rarely consider the impact of multimorbidity. As well as medical self-management, patients (and their carers) need advice to support the psychosocial challenges of living with the chronic disabilities of COPD, and healthcare professionals must be skilled to elicit and respond to the patients' perceptions and priorities [28]. Healthcare systems must adapt their services to providing on-going flexible support for self-management within financial and resource constraints. Finally, we need to measure success not only by the health service priority of reducing admissions, but also aspects of general well-being and markers of independent living as we seek to support people living for many years with the disability of COPD and other long-term conditions.

\section{References}

1 Taylor SJC, Pinnock H, Epiphaniou E, et al. A rapid synthesis of the evidence on interventions supporting self-management for people with long-term conditions: PRISMS - Practical Systematic Review of Self-Management Support for long-term conditions. Health Serv Deliv Res 2014; 2: 53.

2 Gadoury MA, Schwartzman K, Rouleau M, et al. Self-management reduces both short- and long-term hospitalisation in COPD. Eur Respir J 2005; 26: 853-857.

3 Bourbeau J, Julien M, Maltais F, et al. Reduction of hospital utilization in patients with chronic obstructive pulmonary disease: a disease-specific self-management intervention. Arch Intern Med 2003; 163: 585-591.

4 Rice KL, Dewan N, Bloomfield HE, et al. Disease management program for chronic obstructive pulmonary disease: a randomized controlled trial. Am J Respir Crit Care Med 2010; 182: 890-896.

5 Bischoff EW, Akkermans R, Bourbeau J, et al. Comprehensive self management and routine monitoring in chronic obstructive pulmonary disease patients in general practice: randomised controlled trial. BMJ 2012; 345: e7642.

6 Bucknall CE, Miller G, Lloyd SM, et al. Glasgow supported self-management trial (GSuST) for patients with moderate to severe COPD: randomised controlled trial. BMJ 2012; 344: e1060.

7 Fan VS, Gaziano JM, Lew R, et al. A comprehensive care management program to prevent chronic obstructive pulmonary disease hospitalizations: a randomized, controlled trial. Ann Intern Med 2012; 156: 673-683.

8 Zwerink M, Brusse-Keizer M, van der Valk PD, et al. Self management for patients with chronic obstructive pulmonary disease. Cochrane Database Syst Rev 2014; 3: CD002990.

9 Jordan RE, Majothi S, Heneghan NR, et al. Supported self-management for patients with moderate to severe chronic obstructive pulmonary disease (COPD): an evidence synthesis and economic analysis. Health Technol Assess 2015; 19: 36.

10 Jonkman NH, Westland H, Trappenburg JCA, et al. Characteristics of effective self-management interventions in patients with COPD: individual patient data meta-analysis. Eur Respir J 2016; 48: 55-68.

11 Ure J, Hanley J, Pinnock H, et al. Piloting tele-monitoring in chronic obstructive pulmonary disease: a mixed methods exploration of issues in design and implementation. Prim Care Respir J 2012; 21: 57-64.

12 Williams V, Hardinge M, Ryan S, et al. Patients' experience of identifying and managing exacerbations in COPD: a qualitative study. NPJ Prim Care Respir Med 2014; 24: 14062.

13 Burton C, Pinnock H, McKinstry B. Changes in physiological measures with symptoms and decision to treat exacerbations of chronic obstructive pulmonary disease: analysis of telemonitoring data. J Telemed Telecare 2015; 21: 29-36.

14 Calverley P, Pauwels R, Lofdahl CG, et al. Relationship between respiratory symptoms and medical treatment in exacerbations of COPD. Eur Respir J 2005; 26: 406-413.

15 Adams K, Greiner AC, Corrigan JM, eds. 1st Annual Crossing the Quality Chasm Summit: A Focus on Communities. Washington, The National Academic Press, 2004; pp. 57-66.

16 Parke HL, Epiphaniou E, Pearce G, et al. Self-management support interventions for stroke survivors: a systematic meta-review. PLoS One 2015; 10: e0131448.

17 Pearce G, Parke H, Pinnock H, et al. The PRISMS taxonomy of self-management support: derivation of a novel taxonomy and initial testing of utility. J Health Serv Res Policy 2016; 21: 73-82.

18 Pinnock H, Kendall M, Murray S, et al. Living and dying with severe chronic obstructive pulmonary disease: multi-perspective longitudinal qualitative study. BMJ 2011; 342: d142.

19 Kendall M, Buckingham S, Ferguson S, et al. Exploring the concept of need in people with very severe chronic obstructive pulmonary disease: a qualitative study. BMJ Support Palliat Care 2015; [in press DOI: 10.1136/ bmjspcare-2015-000904]. 
20 Kaptein AA, Sharloo M, Fischer MJ, et al. Illness perceptions and COPD: an emerging field for COPD patient management. J Asthma 2008; 45: 625-629.

21 MacNab M, Lee SH, McCloughan L, et al. Oximetry-supported self-management for chronic obstructive pulmonary disease: mixed method evaluation of a pilot project. BMC Health Serv Res 2015; 15: 485.

22 Barnett K, Mercer SW, Norbury M, et al. Epidemiology of multimorbidity and implications for health care, research, and medical education: a cross-sectional study. Lancet 2012; 380: 37-43.

23 Pinnock H, Epiphaniou E, Pearce G, et al. Implementing supported self-management for asthma: a systematic review of implementation studies. BMC Med 2015; 13: 127.

24 Buckingham S, Kendall M, Ferguson S, et al. HELPing older people with very severe chronic obstructive pulmonary disease (HELP-COPD): piloting a practical intervention. NPJ Prim Care Respir Med 2015; 25: 15020.

25 Kielmann T, Huby G, Powell A, et al. From support to boundary: a qualitative study of the border between self-care and professional care. Patient Educ Couns 2010; 79: 55-61.

26 Fairbrother P, Pinnock H, Hanley J, et al. Continuity, but at what cost? The impact of telemonitoring COPD on continuities of care: a qualitative study. Prim Care Respir J 2012; 21: 322-328.

27 Taylor SJ, Sohanpal R, Bremner SA, et al. Self-management support for moderate-to-severe chronic obstructive pulmonary disease: a pilot randomised controlled trial. Br J Gen Pract 2012; 62: e687-e695.

28 Kaptein AA Fischer MJ, Sharloo M. Self-management in patients with COPD: theoretical context, content, outcomes and integration into clinical care. Int J COPD 2014; 9: 907-917. 